Article

\title{
Review of the Life Cycle Greenhouse Gas Emissions from Different Photovoltaic and Concentrating Solar Power Electricity Generation Systems
}

\author{
Raghava Kommalapati $^{1,2, *}$, Akhil Kadiyala ${ }^{1}$, Md. Tarkik Shahriar ${ }^{1,2}$ and Ziaul Huque ${ }^{3}$ \\ 1 Center for Energy \& Environmental Sustainability, Prairie View A\&M University, Prairie View, TX 77446, \\ USA; akhilkadiyala@gmail.com (A.K.); mshahriar@pvamu.edu (M.T.S.) \\ 2 Department of Civil \& Environmental Engineering, Prairie View A\&M University, Prairie View, \\ TX 77446, USA \\ 3 Department of Mechanical Engineering, Prairie View A\&M University, Prairie View, TX 77446, USA; \\ zihuque@pvamu.edu \\ * Correspondence: rrkommalapati@pvamu.edu; Tel.: +1-936-261-1660
}

Academic Editor: Sergio Ulgiati

Received: 8 October 2016; Accepted: 6 March 2017; Published: 11 March 2017

\begin{abstract}
This paper contains an extensive review of life cycle assessment (LCA) studies on greenhouse gas emissions (GHG) from different material-based photovoltaic (PV) and working mechanism-based concentrating solar power (CSP) electricity generation systems. Statistical evaluation of the life cycle GHG emissions is conducted to assess the role of different PVs and CSPs in reducing GHG emissions. The widely-used parabolic trough and central receiver CSP electricity generation systems emitted approximately 50\% more GHGs than the paraboloidal dish, solar chimney, and solar pond CSP electricity generation systems. The cadmium telluride PVs and solar pond CSPs contributed to minimum life cycle GHGs. Thin-film PVs are also suitable for wider implementation, due to their lower Energy Pay-Back Time (EPBT) periods, in addition to lower GHG emission, in comparison with c-Si PVs.
\end{abstract}

Keywords: life cycle assessment; greenhouse gas emissions; solar energy; photovoltaics; concentrating solar power; electricity generation

\section{Introduction}

Solar energy may be defined as the energy harnessed from the solar radiation reaching the Earth's surface. Solar energy may be harnessed for electricity generation by using (a) photovoltaic (PV) and (b) concentrating solar power (CSP) systems. The PV systems work on the principle of direct conversion of solar radiation into electricity when sunlight comes in contact with materials (e.g., semiconductors) exhibiting the photoelectric effect (where there is a release of electrons after absorption of photons of light). A combination of multiple PV modules (a combination of a number of solar cells connected to each other and mounted over a frame) is generally referred to as an array. The PV modules or arrays may be connected in series or parallel to generate electricity. The CSP systems accumulate the sun's energy to a receiver that serves as a heat source to be used, subsequently, in moving steam or wind turbines to generate electricity. An intermediate medium (referred to as the heat transfer fluid) is used for the transfer of thermal energy to generate electricity with the case of CSP being located at a place different from the location of the receiver.

The total electricity generation in 2012 across the world was reported to be 21.53 trillion $\mathrm{kWh}$ [1]. The projected world electricity generation for 2040 is 39 trillion $\mathrm{kWh}$ (an increase of $81 \%$ from 2012) [2]. Renewable energy sources have been projected to account for 9.6 trillion $\mathrm{kWh}(25 \%)$ of the world's 
total electricity generation in 2040. With the continuing depletion of traditional non-renewable energy sources, the necessity for generating electricity through the use of renewable energy sources (solar, hydro, wind, biomass, geothermal) increased manifold. Solar energy accounted for only 0.096 trillion $\mathrm{kWh}(0.44 \%)$ of the world's total electricity generated in 2012. Based on the 2012 statistics, solar energy was the fourth largest renewable energy source for electricity generation after hydro (3.646 trillion $\mathrm{kWh}$ ), wind ( 0.52 trillion $\mathrm{kWh}$ ), and biomass (0.384 trillion $\mathrm{kWh}$ ) [1]. These statistics indicate that there is ample scope to generate electricity on a large scale using solar energy. In the United States of America (USA) alone, solar energy-based electricity generation is projected to increase from 8 gigawatts (GW) in 2012 to $48 \mathrm{GW}$ in 2040. Increasing the contribution of renewables, like solar, is imperative to lower global GHG emissions and meet climate goals [3].

Under such a projected increase, one needs to evaluate the sustainability of different types of PVs and CSPs by analyzing life cycle greenhouse gas (GHG) emissions resulting from their adoption. The life cycle assessment (LCA) approach helps evaluate the net GHG emissions resulting from the use of solar energy as a fuel. LCA is an analytical method that provides an assessment of the environmental impacts of the considered products and technologies from a 'cradle to grave' systems perspective utilizing the detailed input and output parameters that operate within the designated system boundaries.

Many studies analyzed the LCA of PVs [4-34] and CSPs [35-46]. The use of PV/CSP electricity generation systems around the world is being encouraged in view of the advantages that solar energy is a free and abundant resource from which electricity may be generated with relatively low operational and maintenance costs in comparison with other renewable energy sources. The use of PV/CSP electricity generation systems is inhibited by issues such as the intermittency and the unpredictability of solar radiation on cloudy days/nights and the requirement of large areas of land for utility-scale CSP installation. A more detailed description of the LCA boundary conditions, greenhouse gas (GHG) emissions, and site-specific characteristics associated with each of the aforementioned PV and CSP electricity generation system studies is provided in the Sections 3.1 and 3.2.

The majority of the PV and CSP LCA publications to date have emphasized on the determination of the life cycle GHG emissions from select PV/CSP electricity generation systems. There were limited studies that analyzed the life cycle GHG emissions from a broader spectrum of PV [47,48] and CSP [48] electricity generation systems. None of the earlier studies examined the life cycle GHG emissions and compared them across all off the currently available distinct PV and CSP electricity generation system types. This study aims to fill this knowledge gap by performing a comprehensive review of the literature on all the currently available PV and CSP LCA studies, followed by a statistical evaluation of the life cycle GHG emissions from the reviewed PV and CSP electricity generation systems individually. The results from the statistical evaluation of the life cycle GHG emissions will assist energy policy-makers and environmental professionals in decision-making and selection of sustainable solar solutions to power production.

\section{Methodology}

A review of the literature showed that the PV and CSP electricity generation systems may further be categorized on the basis of material type and working mechanism governing the accumulation of solar energy, respectively. The different categories of PV electricity generation systems [49] are as follows:

- Non-organic material-based PVs:as follows:

$>\quad$ Crystalline-silicon (c-Si): light is allowed to filter through a series of layers comprising of a protective glass cover, a transparent adhesive, and an anti-reflective coating material to reach positive- and a negative-type silicon crystalline materials bound together and held with positive and negative electrical contacts. The c-Si cells are referred to as the mono- or single-crystalline silicon (sc-Si) cells, when they are cut from a single high-purity crystal. 
If the c-Si cells are obtained in the form of wafers resulting from the process of cooling and solidification of molten silicon crystal blocks, then, they are designated as poly- or multi-crystalline silicon (mc-Si) cells.

> Thin-films: light is allowed to filter through a series of layers comprising a transparent coating, an anti-reflective layer, positive and negative semi-conductor materials, a contact plate and a substrate. Thin films may further be categorized as amorphous-silicon (a-Si), multi-junction thin-film silicon ( $\mu \mathrm{c}-\mathrm{Si})$, cadmium telluride (CdTe), copper-indiumdiselenide (CIS), and copper-indium-gallium-diselenide (CIGS) thin films on the basis of the material components used.

- Organic material based PVs:

$>\quad$ Organic PVs (OPV): built from thin films of organic semiconductors that include polymers.

> Dye-sensitized solar cells (DSSC): consists of a photosensitive dye and is based on a semiconductor formed between a photo-sensitized anode and an electrolyte that facilitates the movement of electrons to generate electricity

The different categories of CSP electricity generation systems [48] are as follows:

- Parabolic trough: arrays of parabolic trough reflectors reflect the sunlight to a black absorber tube that is cooled by a heat-transferring fluid. The heat-transferring fluid when hot, is pumped to the heat exchanger of a steam Rankine cycle for power generation.

- Central receiver: solar radiation is reflected on to a centrally placed receiver mounted over the top of a tower by a collector that comprises of two large heliostats.

- Paraboloidal dish: a paraboloidal dish reflector is used as a solar collector and the heat to electricity conversion is achieved by using a Stirling engine.

- Solar chimney: a flat area is covered by a glass cover (with soil and air underneath) that is inclined toward the middle, where a chimney is located at the center and is exposed to the sun. The hot air rising up through the chimney generates electricity by using a wind turbine.

- Solar pond: a large reservoir of water with a black bottom absorbs solar radiation and transforms it into heat in the form of hot water.

This study adopted the same classification of PVs (sc-Si, mc-Si, a-Si, $\mu c-S i, C d T e, C I S, C I G S, O P V$, DSSC) and CSPs (parabolic trough, central receiver, paraboloidal dish, solar chimney, solar pond) on the basis of the classifications proposed by the IPCC Report [49] and Amponsah et al. [48], respectively, to evaluate the life cycle GHG emissions from using different PV and CSP electricity generation systems. Each of the reviewed PV and CSP LCA study was first assigned a distinct category. Next, the life cycle GHG emissions from material-based PV and working mechanism-based CSP electricity generation systems were evaluated using statistical metrics (sample size, mean, standard deviation, minimum, maximum, standard error of the mean, quartile 1, quartile 2 or median, quartile 3) and graphical representations (error bars representing the mean with $95 \%$ confidence intervals, box plots representing the quartiles with outliers). While the error bars demonstrate the degree of confidence in the mean GHG emissions, the box plots provide information on the degree of variation among the LCA studies characterized by different PV and CSP categories.

\section{Results and Discussion}

\subsection{Review of PV LCA Studies}

There are numerous studies [4-34] that evaluated the life cycle environmental impacts of using PVs for electricity generation. One needs to define the system boundary conditions (that includes details on the activities or processes to be considered in the analysis) and a functional unit of measure 
(that enables quantification of the net environmental impacts from carrying out an activity or a process as defined within the LCA system boundary conditions) when performing a LCA.

The majority of the aforementioned studies $[6,8,13,17-19,22,23,28,30-32,34]$ that performed the LCA of PV electricity generation systems defined the system boundary conditions to include activities such as raw material extraction, manufacturing processes, transport, installation, operation, maintenance, and end-of-life processes (dismantling, recycling, and final disposal). Some studies $[11,16,29]$ excluded the consideration of end-of-life processes to define their system boundaries to be limited to raw material extraction, manufacturing processes, transport, installation, operation, and maintenance. The remaining studies $[4,5,7,9,10,12,14,15,18,20,21,24-27,33]$ limited the system boundaries to PV module production.

The common functional unit of measure adopted by the majority of the PV LCA studies is grams of carbon dioxide equivalent per kilowatt hour $\left(\mathrm{gCO}_{2} \mathrm{e} / \mathrm{kWh}\right)$ of electricity produced. Accordingly, this study also adopts the functional unit of measure for GHG emissions to be $\mathrm{gCO}_{2} \mathrm{e} / \mathrm{kWh}$ of electricity produced.

Table 1 provides a summary of the PV module categorization (based on the type of material) along with the corresponding GHG emissions (in $\mathrm{gCO}_{2} \mathrm{e} / \mathrm{kWh}$ ) and energy payback time (EPBT, expressed in years) periods for each of the reviewed PV LCA study. Table 1 also provides additional site-specific details that included module efficiency $\left(\eta_{1}\right.$, expressed in \%), performance ratio $\left(\eta_{2}\right.$, expressed in \%), power rating ( $\mathrm{PR}$, expressed in $\mathrm{kW})$, available solar radiation at the location (SR, expressed in $\mathrm{kWh} / \mathrm{m}^{2} / \mathrm{yr}$ ), the type of installation (TI: roof-top/ground-mount/building-integrated with the angle of tilt), and the geographical location (GL) for the installed PV systems. The EPBT may be defined as the time period for which a PV system should operate to recover an equivalent amount of energy spent in the production of the installed PV system. $\eta_{1}$ provides a measure of the performance of the PV module in generating energy from sunlight and is defined as the ratio of energy output from the PV module to the input energy from the sun. $\eta_{2}$ is defined as the ratio of the actual and theoretically possible energy outputs and may be used in comparing the PV systems at different locations across the world (considering that $\eta_{2}$ is independent of the orientation and the amount of incident solar radiation). Based on the review of $31 \mathrm{PV}$ electricity generation LCA studies (refer to Table 1$)$, one may note that mc-Si $(N=35) \mathrm{PV}$ electricity generation systems were more in number compared to sc-Si $(N=24), \operatorname{CdTe}(N=21)$, a-Si $(N=16), \operatorname{CIS}(N=3), \operatorname{DSSC}(N=2), \mu \mathrm{c}-\mathrm{Si}(N=1)$, and CIGS $(N=1)$ PV electricity generation systems. There were no LCA studies on the use of OPVs in electricity generation.

Table 1. Greenhouse Gas (GHG) emissions and Energy Pay-Back Time (EPBT) periods for photovoltaic (PV) electricity generation systems.

\begin{tabular}{|c|c|c|c|c|}
\hline Source & $\begin{array}{c}\text { PV Category } \\
\text { (Supplementary Case } \\
\text { Description) }\end{array}$ & $\begin{array}{c}\text { GHG } \\
\text { Emissions } \\
\left(\mathrm{gCO}_{2} \mathrm{e} / \mathrm{kWh}\right)\end{array}$ & $\begin{array}{l}\text { EPBT } \\
\text { (Years) }\end{array}$ & $\begin{array}{c}\text { Additional Features } \\
\eta_{1}(\%), \eta_{2}(\%), \text { PR }(\mathrm{kW}), \\
\text { SR }\left(\mathrm{kWh} / \mathrm{m}^{2} / \mathrm{yr}\right), \mathrm{TI}, \mathrm{GL}\end{array}$ \\
\hline \multirow{4}{*}{$\begin{array}{l}\text { Schaefer and } \\
\text { Hagedorn [4] }\end{array}$} & $\begin{array}{l}\text { sc-Si (annual cell production of } \\
2.5 \mathrm{MW} \text { per year; annual load } \\
\text { duration time of } 2000 \mathrm{~h} \text { per year) }\end{array}$ & 130 & 3.7 & $\begin{array}{c}\eta_{1}=14 ; P R=300 \\
\text { GL }=\text { Germany }\end{array}$ \\
\hline & $\begin{array}{l}\text { sc-Si (annual cell production of } \\
2.5 \mathrm{MW} \text { per year; annual load } \\
\text { duration time of } 1000 \mathrm{~h} \text { per year) }\end{array}$ & 250 & 7.3 & $\begin{array}{c}\eta_{1}=14 ; P R=300 \\
\text { GL }=\text { Germany }\end{array}$ \\
\hline & $\begin{array}{l}\text { sc-Si (annual cell production of } \\
25 \mathrm{MW} \text { per year; annual load } \\
\text { duration time of } 2000 \mathrm{~h} \text { per year) }\end{array}$ & 70 & 3.7 & $\begin{array}{c}\eta_{1}=15.5 ; P R=1500 \\
\quad G L=\text { Germany }\end{array}$ \\
\hline & $\begin{array}{l}\text { sc-Si (annual cell production of } \\
25 \mathrm{MW} \text { per year; annual load } \\
\text { duration time of } 1000 \mathrm{~h} \text { per year) }\end{array}$ & 150 & 7.3 & $\begin{array}{c}\eta_{1}=15.5 ; P R=1500 \\
\quad \text { GL }=\text { Germany }\end{array}$ \\
\hline
\end{tabular}


Table 1. Cont

\begin{tabular}{|c|c|c|c|c|}
\hline Source & $\begin{array}{c}\text { PV Category } \\
\text { (Supplementary Case } \\
\text { Description) }\end{array}$ & $\begin{array}{c}\text { GHG } \\
\text { Emissions } \\
\left(\mathrm{gCO}_{2} \mathrm{e} / \mathrm{kWh}\right)\end{array}$ & $\begin{array}{l}\text { EPBT } \\
\text { (Years) }\end{array}$ & $\begin{array}{c}\text { Additional Features } \\
\eta_{1}(\%), \eta_{2}(\%), \text { PR (kW), } \\
\text { SR (kWh/m²/yr), TI, GL }\end{array}$ \\
\hline \multirow{8}{*}{$\begin{array}{l}\text { Schaefer and } \\
\text { Hagedorn [4] }\end{array}$} & $\begin{array}{l}\text { mc-Si (annual cell production of } \\
2.5 \mathrm{MW} \text { per year; annual load } \\
\text { duration time of } 2000 \mathrm{~h} \text { per year) }\end{array}$ & 120 & 3.6 & $\begin{array}{c}\eta_{1}=12 ; P R=300 \\
\text { GL }=\text { Germany }\end{array}$ \\
\hline & $\begin{array}{l}\text { mc-Si (annual cell production of } \\
2.5 \mathrm{MW} \text { per year; annual load } \\
\text { duration time of } 1000 \mathrm{~h} \text { per year) }\end{array}$ & 250 & 7.1 & $\begin{array}{c}\eta_{1}=12 ; P R=300 \\
\text { GL }=\text { Germany }\end{array}$ \\
\hline & $\begin{array}{l}\text { mc-Si (annual cell production of } \\
25 \mathrm{MW} \text { per year; annual load } \\
\text { duration time of } 2000 \mathrm{~h} \text { per year) }\end{array}$ & 50 & 3.6 & $\begin{array}{l}\eta_{1}=13.5 ; P R=1500 \\
\quad G L=\text { Germany }\end{array}$ \\
\hline & $\begin{array}{l}\text { mc-Si (annual cell production of } \\
25 \mathrm{MW} \text { per year; annual load } \\
\text { duration time of } 1000 \mathrm{~h} \text { per year) }\end{array}$ & 110 & 7.1 & $\begin{array}{c}\eta_{1}=13.5 ; P R=1500 \\
\quad G L=\text { Germany }\end{array}$ \\
\hline & $\begin{array}{l}\text { a-Si (annual cell production of } \\
2.5 \mathrm{MW} \text { per year; annual load } \\
\text { duration time of } 2000 \mathrm{~h} \text { per year) }\end{array}$ & 90 & 2.9 & $\begin{array}{l}\eta_{1}=6 ; P R=300 \\
\text { GL }=\text { Germany }\end{array}$ \\
\hline & $\begin{array}{l}\text { a-Si (annual cell production of } \\
2.5 \mathrm{MW} \text { per year; annual load } \\
\text { duration time of } 1000 \mathrm{~h} \text { per year) }\end{array}$ & 170 & 5.8 & $\begin{array}{l}\eta_{1}=6 ; P R=300 \\
\text { GL }=\text { Germany }\end{array}$ \\
\hline & $\begin{array}{l}\text { a-Si (annual cell production of } \\
25 \mathrm{MW} \text { per year; annual load } \\
\text { duration time of } 2000 \mathrm{~h} \text { per year) }\end{array}$ & 50 & 2.9 & $\begin{array}{c}\eta_{1}=8 ; P R=1500 \\
\text { GL }=\text { Germany }\end{array}$ \\
\hline & $\begin{array}{l}\text { a-Si (annual cell production of } \\
25 \mathrm{MW} \text { per year; annual load } \\
\text { duration time of } 1000 \mathrm{~h} \text { per year) }\end{array}$ & 100 & 5.8 & $\begin{array}{c}\eta_{1}=8 ; P R=1500 \\
G L=\text { Germany }\end{array}$ \\
\hline \multirow{3}{*}{$\begin{array}{l}\text { Netherlands } \\
\text { Agency for Energy } \\
\text { and the } \\
\text { Environment } \\
\text { Report [5] }\end{array}$} & mc-Si (worst case) & 167 & 3.8 & $\begin{array}{c}\eta_{1}=13 ; \eta_{2}=75 ; S R=1000 \\
G L=\text { Netherlands }\end{array}$ \\
\hline & mc-Si (base case) & 31 & 1.3 & $\begin{array}{c}\eta_{1}=16 ; \eta_{2}=80 ; S R=1000 \\
G L=\text { Netherlands }\end{array}$ \\
\hline & mc-Si (best case) & 9.8 & 0.5 & $\begin{array}{c}\eta_{1}=18 ; \eta_{2}=85 ; S R=1000 \\
\quad G L=\text { Netherlands }\end{array}$ \\
\hline Nieuwlaar et al. [6] & $\mathrm{a}-\mathrm{Si}$ & 47 & 4 & $\begin{array}{l}\eta_{1}=10 ; \mathrm{TI}=\text { roof-top; } \\
\mathrm{GL}=\text { Netherlands }\end{array}$ \\
\hline \multirow{5}{*}{ Kato et al. [7] } & sc-Si (worst case) & 91 & 15.5 & $\begin{array}{l}\eta_{2}=81 ; \mathrm{PR}=3 ; \mathrm{SR}=1427 ; \\
\mathrm{TI}=\text { roof-top; } \mathrm{GL}=\text { Japan }\end{array}$ \\
\hline & sc-Si (base case) & 65 & 11 & $\begin{array}{l}\eta_{2}=81 ; P R=3 ; S R=1427 ; \\
T I=\text { roof-top; } \mathrm{GL}=\text { Japan }\end{array}$ \\
\hline & sc-Si (optimistic case) & 21 & 4 & $\begin{array}{l}\eta_{2}=81 ; \mathrm{PR}=3 ; \mathrm{SR}=1427 \\
\mathrm{TI}=\text { roof-top; } \mathrm{GL}=\text { Japan }\end{array}$ \\
\hline & mc-Si & 18 & 2.5 & $\begin{array}{l}\eta_{2}=81 ; \mathrm{PR}=3 ; \mathrm{SR}=1427 \\
\mathrm{TI}=\text { roof-top; } \mathrm{GL}=\text { Japan }\end{array}$ \\
\hline & $\mathrm{a}-\mathrm{Si}$ & 15 & 1.5 & $\begin{array}{l}\eta_{2}=81 ; \mathrm{PR}=3 ; \mathrm{SR}=1427 ; \\
\mathrm{TI}=\text { roof-top; } \mathrm{GL}=\text { Japan }\end{array}$ \\
\hline \multirow{2}{*}{$\begin{array}{l}\text { Dones and } \\
\text { Frischknecht [8] }\end{array}$} & mc-Si & 189 & NA & $\begin{array}{c}\eta_{1}=14 ; \mathrm{PR}=3 ; \\
\mathrm{TI}=\text { roof-top; } \mathrm{GL}=\text { Switzerland }\end{array}$ \\
\hline & sc-Si & 114 & NA & $\begin{array}{c}\eta_{1}=16.5 ; \mathrm{PR}=3 ; \\
\mathrm{TI}=\text { roof-top; } \mathrm{GL}=\text { Switzerland }\end{array}$ \\
\hline Frankl et al. [9] & sc-Si & 200 & 9 & $\begin{array}{c}\eta_{1}=11.2 ; \mathrm{PR}=20 ; \\
\mathrm{SR}=1700 ; \mathrm{TI}=\text { roof-top }, 30^{\circ} \text { tilt } ; \\
\mathrm{GL}=\text { Italy }\end{array}$ \\
\hline
\end{tabular}


Table 1. Cont

\begin{tabular}{|c|c|c|c|c|}
\hline Source & $\begin{array}{c}\text { PV Category } \\
\text { (Supplementary Case } \\
\text { Description) }\end{array}$ & $\begin{array}{c}\text { GHG } \\
\text { Emissions } \\
\left(\mathrm{gCO}_{2} \mathrm{e} / \mathrm{kWh}\right)\end{array}$ & $\begin{array}{l}\text { EPBT } \\
\text { (Years) }\end{array}$ & $\begin{array}{c}\text { Additional Features } \\
\eta_{1}(\%), \eta_{2}(\%), \text { PR }(\mathrm{kW}), \\
\text { SR }\left(\mathrm{kWh} / \mathrm{m}^{2} / \mathrm{yr}\right), \mathrm{TI}, \mathrm{GL}\end{array}$ \\
\hline \multirow{9}{*}{ Kato et al. [10] } & sc-Si (worst case) & 83 & 11.8 & $\begin{array}{c}\eta_{1}=12.2 ; \eta_{2}=81 ; P R=3 ; \\
S R=1427 ; T I=\text { roof-top; } \\
\text { GL = Japan }\end{array}$ \\
\hline & sc-Si (base case) & 61 & 8.9 & $\begin{array}{c}\eta_{1}=12.2 ; \eta_{2}=81 ; P R=3 \\
S R=1427 ; T I=\text { roof-top; } \\
\text { GL = Japan }\end{array}$ \\
\hline & sc-Si (best case) & 25 & 3.3 & $\begin{array}{c}\eta_{1}=12.2 ; \eta_{2}=81 ; P R=3 \\
S R=1427 ; T I=\text { roof-top; } \\
\text { GL = Japan }\end{array}$ \\
\hline & $\begin{array}{c}\text { mc-Si (annual cell production of } \\
10 \mathrm{MW} \text { per year) }\end{array}$ & 20 & 2.4 & $\begin{array}{c}\eta_{1}=11.6 ; \eta_{2}=81 ; P R=3 \\
S R=1427 ; T I=\text { roof-top; } \\
\text { GL = Japan }\end{array}$ \\
\hline & $\begin{array}{c}\text { mc-Si (annual cell production of } \\
30 \mathrm{MW} \text { per year) }\end{array}$ & 18 & 2.2 & $\begin{array}{c}\eta_{1}=11.7 ; \eta_{2}=81 ; P R=3 ; \\
S R=1427 ; T I=\text { roof-top; } \\
\text { GL = Japan }\end{array}$ \\
\hline & $\begin{array}{c}\text { mc-Si (annual cell production of } \\
100 \mathrm{MW} \text { per year) }\end{array}$ & 13 & 1.5 & $\begin{array}{c}\eta_{1}=15.7 ; \eta_{2}=81 ; P R=3 ; \\
S R=1427 ; T I=\text { roof-top; } \\
\text { GL = Japan }\end{array}$ \\
\hline & $\begin{array}{l}\text { a-Si (annual cell production of } \\
10 \mathrm{MW} \text { per year) }\end{array}$ & 17 & 2.1 & $\begin{array}{c}\eta_{1}=8 ; \eta_{2}=81 ; P R=3 \\
S R=1427 ; T I=\text { roof-top; } \\
\text { GL = Japan }\end{array}$ \\
\hline & $\begin{array}{l}\text { a-Si (annual cell production of } \\
30 \mathrm{MW} \text { per year) }\end{array}$ & 13 & 1.7 & $\begin{array}{c}\eta_{1}=10 ; \eta_{2}=81 ; P R=3 \\
S R=1427 ; T I=\text { roof-top; } \\
\text { GL = Japan }\end{array}$ \\
\hline & $\begin{array}{l}\text { a-Si (annual cell production of } \\
100 \mathrm{MW} \text { per year) }\end{array}$ & 9 & 1.1 & $\begin{array}{c}\eta_{1}=12 ; \eta_{2}=81 ; \mathrm{PR}=3 \\
\mathrm{SR}=1427 ; \mathrm{TI}=\text { roof-top; } \\
\text { GL = Japan }\end{array}$ \\
\hline Lewis et al. [11] & $\mathrm{a}-\mathrm{Si}$ & 187.8 & 5.14 & $\begin{array}{c}\eta_{1}=3.89 \\
\mathrm{TI}=\text { building-integrated; } \\
\text { GL = USA }\end{array}$ \\
\hline \multirow{3}{*}{ Alsema [12] } & sc-Si & 60 & 3.2 & $\begin{array}{c}\eta_{1}=14 ; \mathrm{SR}=1700 ; \mathrm{TI}=\text { roof-top} ; \\
\mathrm{GL}=\text { Netherlands }\end{array}$ \\
\hline & mc-Si & 50 & 3.2 & $\begin{array}{c}\eta_{1}=13 ; S R=1700 ; \text { TI = roof-top; } \\
\text { GL = Netherlands }\end{array}$ \\
\hline & $\mathrm{a}-\mathrm{Si}$ & 50 & 2.7 & $\begin{aligned} \eta_{1}=7 ; S R & =1700 ; \mathrm{TI}=\text { roof-top; } \\
\mathrm{GL} & =\text { Netherlands }\end{aligned}$ \\
\hline \multirow{2}{*}{$\begin{array}{c}\text { Oliver and Jackson } \\
\text { [13] }\end{array}$} & mc-Si & 120 & NA & $\begin{array}{c}\eta_{1}=14 ; \eta_{2}=55 \\
\mathrm{TI}=\text { building-integrated; } \\
\mathrm{GL}=\text { Swiss Jura Alps, Europe }\end{array}$ \\
\hline & mc-Si & 170 & NA & $\begin{array}{c}\eta_{1}=14 ; \eta_{2}=85 \\
\mathrm{TI}=\text { building-integrated; } \\
\mathrm{GL}=\text { Swiss Jura Alps, Europe }\end{array}$ \\
\hline \multirow{2}{*}{ Greijer et al. [14] } & DSSC & 19 & NA & $\begin{aligned} \eta_{1} & =7 ; \eta_{2}=53 ; S R=2190 \\
G L & =\text { Sahara Desert }, \text { Africa }\end{aligned}$ \\
\hline & DSSC & 47 & NA & $\begin{array}{l}\eta_{1}=12 ; \eta_{2}=53 ; S R=2190 \\
G L=\text { Sahara Desert, Africa }\end{array}$ \\
\hline
\end{tabular}


Table 1. Cont

\begin{tabular}{|c|c|c|c|c|}
\hline \multirow[b]{2}{*}{ Source } & \multirow{2}{*}{$\begin{array}{c}\text { PV Category } \\
\text { (Supplementary Case } \\
\text { Description) }\end{array}$} & \multirow{2}{*}{$\begin{array}{c}\text { GHG } \\
\text { Emissions } \\
\left(\mathrm{gCO}_{2} \mathrm{e} / \mathrm{kWh}\right)\end{array}$} & \multirow[b]{2}{*}{$\begin{array}{l}\text { EPBT } \\
\text { (Years) }\end{array}$} & \multirow{2}{*}{$\begin{array}{c}\text { Additional Features } \\
\eta_{1}(\%), \eta_{2}(\%), \text { PR }(\mathrm{kW}), \\
\text { SR }\left(\mathrm{kWh} / \mathrm{m}^{2} / \mathrm{yr}\right), \mathrm{TI}, \mathrm{GL}\end{array}$} \\
\hline & & & & \\
\hline \multirow{3}{*}{ Kato et al. [15] } & $\begin{array}{c}\text { CdTe (annual cell production of } \\
10 \mathrm{MW} \text { per year) }\end{array}$ & 14 & 1.7 & $\begin{array}{c}\eta_{1}=10.3 ; \eta_{2}=81 ; P R=3 \\
S R=1427 ; T I=\text { roof-top; } \\
\text { GL = Japan }\end{array}$ \\
\hline & $\begin{array}{c}\text { CdTe (annual cell production of } \\
30 \mathrm{MW} \text { per year) }\end{array}$ & 11.5 & 1.4 & $\begin{array}{c}\eta_{1}=11.2 ; \eta_{2}=81 ; P R=3 \\
S R=1427 ; T I=\text { roof-top; } \\
\text { GL = Japan }\end{array}$ \\
\hline & $\begin{array}{c}\text { CdTe (annual cell production of } \\
100 \mathrm{MW} \text { per year) }\end{array}$ & 8.9 & 1.1 & $\begin{array}{c}\eta_{1}=12.4 ; \eta_{2}=81 ; \mathrm{PR}=3 \\
\mathrm{SR}=1427 ; \mathrm{TI}=\text { roof-top; } \\
\mathrm{GL}=\text { Japan }\end{array}$ \\
\hline \multirow{2}{*}{ Nomura et al. [16] } & mc-Si & 104 & NA & GL = Japan \\
\hline & mc-Si & 133 & NA & GL = Japan \\
\hline Meier [17] & $\mathrm{a}-\mathrm{Si}$ & 39 & 4.9 & $\begin{array}{c}\eta_{1}=5.7 \\
\mathrm{TI}=\text { building-integrated; } \\
\text { GL = USA }\end{array}$ \\
\hline Ito et al. [18] & mc-Si & 12 & 1.7 & $\begin{array}{c}\eta_{1}=12.8 ; \eta_{2}=78 ; P R=1,000,000 \\
\left.S R=1854 \text { ( } 10^{\circ} \text { tilt }\right)-2037 \\
\left(40^{\circ} \text { tilt }\right) ; \mathrm{TI}=\text { ground-mount; } \\
\text { GL = Gobi Desert, China }\end{array}$ \\
\hline $\begin{array}{l}\text { Fthenakis and Kim } \\
\text { [19] }\end{array}$ & CdTe & 23.6 & 1.2 & $\begin{array}{c}\eta_{1}=9 ; \eta_{2}=80 ; \mathrm{PR}=25,000 \\
\mathrm{SR}=1800 ; \mathrm{TI}=\text { ground-mount; } \\
\mathrm{GL}=\text { USA }\end{array}$ \\
\hline \multirow{3}{*}{ Alsema et al. [20] } & sc-Si & 35 & 2.6 & $\begin{array}{l}\eta_{1}=14 ; \eta_{2}=75 ; S R=1700 \\
T I=\text { roof-top } ; \mathrm{GL}=\text { Europe }\end{array}$ \\
\hline & mc-Si & 32 & 1.9 & $\begin{array}{c}\eta_{1}=13.2 ; \eta_{2}=75 ; S R=1700 \\
T I=\text { roof-top; } \mathrm{GL}=\text { Europe }\end{array}$ \\
\hline & CdTe & 25 & 1.1 & $\begin{array}{c}\eta_{1}=9 ; \eta_{2}=75 ; S R=1700 ; \\
\text { TI = ground-mount; } \\
\text { GL = Europe }\end{array}$ \\
\hline \multirow{3}{*}{$\begin{array}{l}\text { Fthenakis and } \\
\text { Alsema [21] }\end{array}$} & mc-Si & 37 & 2.2 & $\begin{array}{l}\eta_{1}=13.2 ; \eta_{2}=75 ; S R=1700 \\
T I=\text { roof-top; } G L=\text { Europe }\end{array}$ \\
\hline & CdTe & 21 & 1 & $\begin{array}{l}\eta_{1}=8 ; \eta_{2}=75 ; \mathrm{SR}=1700 \\
\mathrm{TI}=\text { roof-top; } \mathrm{GL}=\text { Europe }\end{array}$ \\
\hline & CdTe & 25 & 1.1 & $\begin{array}{c}\eta_{1}=9 ; \eta_{2}=75 ; S R=1700 \\
\quad T I=\text { roof-top; } \mathrm{GL}=\mathrm{US}\end{array}$ \\
\hline Kannan et al. [22] & sc-Si & 165 & 4.47 & $\begin{aligned} \eta_{1} & =11.86 ; \mathrm{PR}=2.7 ; \mathrm{SR}=1635 ; \\
\mathrm{TI} & =\text { roof-top; } \mathrm{GL}=\text { Singapore }\end{aligned}$ \\
\hline \multirow{3}{*}{$\begin{array}{l}\text { Fthenakis and Kim } \\
\text { [23] }\end{array}$} & mc-Si & 37 & NA & $\begin{array}{c}\eta_{1}=13.2 ; \eta_{2}=80 ; S R=1700 ; \\
T I=\text { roof-top; } G L=\text { Europe }\end{array}$ \\
\hline & sc-Si & 45 & NA & $\begin{array}{l}\eta_{1}=14 ; \eta_{2}=80 ; S R=1700 \\
T I=\text { roof-top } ; \mathrm{GL}=\text { Europe }\end{array}$ \\
\hline & CdTe & 16 & NA & $\begin{array}{c}\eta_{1}=9 ; \eta_{2}=80 ; \mathrm{SR}=1700 \\
\mathrm{TI}=\text { roof-top; } \mathrm{GL}=\text { Europe }\end{array}$ \\
\hline \multirow[b]{2}{*}{ Pacca et al. [24] } & $\mathrm{a}-\mathrm{Si}$ & 34.3 & 3.2 & $\begin{array}{c}\eta_{1}=6.3 ; \mathrm{SR}=1359 ; \mathrm{TI}=\text { roof-top, } \\
12^{\circ} \text { tilt; } \mathrm{GL}=\text { Michigan, USA }\end{array}$ \\
\hline & mc-Si & 72.4 & 7.4 & $\begin{array}{l}\eta_{1}=12.92 ; \mathrm{SR}=1359 \\
\mathrm{TI}=\text { roof-top, } 12^{\circ} \text { tilt; } \\
\mathrm{GL}=\text { Michigan, } \mathrm{USA}\end{array}$ \\
\hline
\end{tabular}


Table 1. Cont.

\begin{tabular}{|c|c|c|c|c|}
\hline Source & $\begin{array}{c}\text { PV Category } \\
\text { (Supplementary Case } \\
\text { Description) }\end{array}$ & $\begin{array}{c}\text { GHG } \\
\text { Emissions } \\
\left(\mathrm{gCO}_{2} \mathrm{e} / \mathrm{kWh}\right)\end{array}$ & $\begin{array}{l}\text { EPBT } \\
\text { (Years) }\end{array}$ & $\begin{array}{c}\text { Additional Features } \\
\eta_{1}(\%), \eta_{2}(\%), \text { PR (kW), } \\
\text { SR (kWh/m²/yr), TI, GL }\end{array}$ \\
\hline \multirow{5}{*}{ Raugei et al. [25] } & mc-Si (worst case) & 167 & 5.5 & $\begin{array}{l}\eta_{1}=14 ; \eta_{2}=75 ; \mathrm{SR}=1700 \\
\mathrm{TI}=\text { roof-top; } \mathrm{GL}=\text { Europe }\end{array}$ \\
\hline & mc-Si (base case) & 72 & 2.4 & $\begin{array}{l}\eta_{1}=14 ; \eta_{2}=75 ; \mathrm{SR}=1700 ; \\
\mathrm{TI}=\text { roof-top; } \mathrm{GL}=\text { Europe }\end{array}$ \\
\hline & mc-Si (best case) & 57 & 2.5 & $\begin{array}{l}\eta_{1}=14 ; \eta_{2}=75 ; \mathrm{SR}=1700 ; \\
\mathrm{TI}=\text { roof-top; } \mathrm{GL}=\text { Europe }\end{array}$ \\
\hline & CIS & 95 & 2.8 & $\begin{array}{l}\eta_{1}=11 ; \eta_{2}=75 ; \mathrm{SR}=1700 ; \\
\mathrm{TI}=\text { roof-top; } \mathrm{GL}=\text { Europe }\end{array}$ \\
\hline & $\mathrm{CdTe}$ & 48 & 1.5 & $\begin{array}{l}\eta_{1}=9 ; \eta_{2}=75 ; S R=1700 \\
T I=\text { roof-top; } G L=\text { Europe }\end{array}$ \\
\hline \multirow{8}{*}{ Fthenakis et al. [26] } & $\begin{array}{l}\text { sc-Si (CrystalClear project with } \\
\text { Ecoinvent database) }\end{array}$ & 32 & NA & $\begin{array}{c}\eta_{1}=14 ; \eta_{2}=80 ; S R=1700 \\
\text { TI = ground-mount; } \\
\text { GL = Europe }\end{array}$ \\
\hline & $\begin{array}{l}\text { sc-Si (UCTE grid mixture with } \\
\text { Ecoinvent database) }\end{array}$ & 42 & NA & $\begin{array}{c}\eta_{1}=14 ; \eta_{2}=80 ; S R=1700 ; \\
\text { TI = ground-mount; } \\
\text { GL = Europe }\end{array}$ \\
\hline & $\begin{array}{l}\text { sc-Si (US grid mixture with } \\
\text { Franklin database) }\end{array}$ & 52 & NA & $\begin{array}{c}\eta_{1}=14 ; \eta_{2}=80 ; S R=1700 \\
\text { TI = ground-mount; } \\
\text { GL = USA }\end{array}$ \\
\hline & $\begin{array}{c}\text { mc-Si (CrystalClear project with } \\
\text { Ecoinvent database) }\end{array}$ & 31 & NA & $\begin{array}{c}\eta_{1}=13.2 ; \eta_{2}=80 ; S R=1700 ; \\
\text { TI = ground-mount; } \\
\text { GL = Europe }\end{array}$ \\
\hline & $\begin{array}{l}\text { mc-Si (UCTE grid mixture with } \\
\text { Ecoinvent database) }\end{array}$ & 41 & NA & $\begin{array}{c}\eta_{1}=13.2 ; \eta_{2}=80 ; S R=1700 \\
\text { TI = ground-mount; } \\
\text { GL = Europe }\end{array}$ \\
\hline & $\begin{array}{l}\text { mc-Si (US grid mixture with } \\
\text { Franklin database) }\end{array}$ & 51 & NA & $\begin{array}{c}\eta_{1}=13.2 ; \eta_{2}=80 ; S R=1700 ; \\
T I=\text { ground-mount; } \\
\text { GL = USA }\end{array}$ \\
\hline & $\begin{array}{l}\text { CdTe (UCTE grid mixture with } \\
\text { Ecoinvent database) }\end{array}$ & 20 & NA & $\begin{array}{c}\eta_{1}=9 ; \eta_{2}=80 ; \mathrm{SR}=1700 \\
\mathrm{TI}=\text { roof-top; } \mathrm{GL}=\text { Europe }\end{array}$ \\
\hline & $\begin{array}{l}\text { CdTe (US grid mixture with } \\
\text { Franklin database) }\end{array}$ & 26 & NA & $\begin{array}{c}\eta_{1}=9 ; \eta_{2}=80 ; S R=1700 \\
T I=\text { roof-top; } G L=U S A\end{array}$ \\
\hline \multirow{4}{*}{ Ito et al. [27] } & mc-Si & 12.1 & 1.9 & $\begin{array}{c}\eta_{1}=12.8 ; \eta_{2}=78 ; \mathrm{PR}=100,000 \\
\mathrm{SR}=1702 \text { (horizontal)-2017 } \\
\left(30^{\circ} \text { tilt); TI = ground-mount; }\right. \\
\text { GL = Gobi Desert, China }\end{array}$ \\
\hline & mc-Si & 9.4 & 1.5 & $\begin{array}{c}\eta_{1}=15.8 ; \eta_{2}=78 ; P R=100,000 \\
S R=1702 \text { (horizontal)-2017 } \\
\left(30^{\circ} \text { tilt); TI = ground-mount; }\right. \\
\text { GL = Gobi Desert, China }\end{array}$ \\
\hline & $\mathrm{a}-\mathrm{Si}$ & 15.6 & 2.5 & $\begin{array}{c}\eta_{1}=6.9 ; \eta_{2}=77.1 ; P R=100,000 \\
\text { SR = } 1702 \text { (horizontal)-2017 } \\
\left(30^{\circ} \text { tilt }\right) ; \mathrm{TI}=\text { ground-mount; } \\
\text { GL = Gobi Desert, China }\end{array}$ \\
\hline & CdTe & 12.8 & 1.9 & $\begin{array}{c}\eta_{1}=9 ; \eta_{2}=77.2 ; P R=100,000 ; \\
S R=1702 \text { (horizontal)-2017 } \\
\left(30^{\circ} \text { tilt); TI = ground-mount; }\right. \\
\text { GL = Gobi Desert, China }\end{array}$ \\
\hline
\end{tabular}


Table 1. Cont

\begin{tabular}{|c|c|c|c|c|}
\hline Source & $\begin{array}{c}\text { PV Category } \\
\text { (Supplementary Case } \\
\text { Description) }\end{array}$ & $\begin{array}{c}\text { GHG } \\
\text { Emissions } \\
\left(\mathrm{gCO}_{2} \mathrm{e} / \mathrm{kWh}\right)\end{array}$ & $\begin{array}{l}\text { EPBT } \\
\text { (Years) }\end{array}$ & $\begin{array}{c}\text { Additional Features } \\
\eta_{1}(\%), \eta_{2}(\%), \text { PR }(\mathrm{kW}), \\
\text { SR }\left(\mathrm{kWh} / \mathrm{m}^{2} / \mathrm{yr}\right), \mathrm{TI}, \mathrm{GL}\end{array}$ \\
\hline Ito et al. [27] & CIS & 10.5 & 1.6 & $\begin{array}{c}\eta_{1}=11 ; \eta_{2}=77.6 ; \mathrm{PR}=100,000 \\
\text { SR }=1702 \text { (horizontal)-2017 } \\
\left(30^{\circ} \text { tilt); TI = ground-mount; }\right. \\
\text { GL = Gobi Desert, China }\end{array}$ \\
\hline $\begin{array}{l}\text { García-Valverde } \\
\text { et al. [28] }\end{array}$ & sc-Si & 131 & 9.08 & $\begin{array}{c}\eta_{2}=62 ; \mathrm{PR}=4.24 ; \mathrm{SR}=1932 \\
\mathrm{TI}=\text { roof-top, } 30^{\circ} \text { tilt; } \\
\text { GL }=\text { Murcia, Spain }\end{array}$ \\
\hline \multirow{5}{*}{ Ito et al. [29] } & sc-Si & 51 & 2.5 & $\begin{array}{c}\eta_{2}=78 ; \mathrm{SR}=1702 ; \\
\mathrm{TI}=\text { ground-mount; } \\
\mathrm{GL}=\text { Gobi Desert, China }\end{array}$ \\
\hline & mc-Si & 42 & 2 & $\begin{array}{c}\eta_{2}=78 ; \mathrm{SR}=1702 ; \\
\mathrm{TI}=\text { ground-mount; } \\
\mathrm{GL}=\text { Gobi Desert, China }\end{array}$ \\
\hline & $\mathrm{a}-\mathrm{Si}$ & 43 & 2.1 & $\begin{array}{c}\eta_{2}=78 ; \mathrm{SR}=1702 ; \\
\mathrm{TI}=\text { ground-mount; } \\
\mathrm{GL}=\text { Gobi Desert } \text { China }\end{array}$ \\
\hline & CIS & 46 & 1.8 & $\begin{array}{c}\eta_{2}=78 ; \mathrm{SR}=1702 ; \\
\mathrm{TI}=\text { ground-mount; } \\
\text { GL = Gobi Desert, China }\end{array}$ \\
\hline & CdTe & 51 & 2.1 & $\begin{array}{c}\eta_{2}=78 ; \mathrm{SR}=1702 ; \\
\mathrm{TI}=\text { ground-mount; } \\
\mathrm{GL}=\text { Gobi Desert, China }\end{array}$ \\
\hline \multirow{3}{*}{ Bravi et al. [30] } & sc-Si & 98.9 & 3.8 & $\begin{array}{c}\eta_{2}=75 ; \mathrm{SR}=1700 ; \mathrm{TI}=\text { roof-top } \\
22^{\circ} \text { tilt; } \mathrm{GL}=\text { Grosseto, Italy }\end{array}$ \\
\hline & mc-Si & 180.3 & 3.5 & $\begin{array}{c}\eta_{2}=75 ; \mathrm{SR}=1700 ; \mathrm{TI}=\text { roof-top } \\
22^{\circ} \text { tilt; } \mathrm{GL}=\text { Grosseto, Italy }\end{array}$ \\
\hline & a-Si, CIGS, CdTe, $\mu \mathrm{c}-\mathrm{Si}$ & 39.2 & 2.5 & $\begin{array}{c}\eta_{2}=75 ; \mathrm{SR}=1700 ; \mathrm{TI}=\text { roof-top } \\
22^{\circ} \text { tilt; } \mathrm{GL}=\text { Grosseto, Italy }\end{array}$ \\
\hline \multirow{3}{*}{$\begin{array}{l}\text { Fthenakis and Kim } \\
\text { [31] }\end{array}$} & sc-Si & 38 & 2.4 & $\begin{array}{c}\eta_{1}=14 ; \eta_{2}=80 ; P R=24 \\
S R=1700-2280 ; T I=\text { roof-top; } \\
\text { GL = Europe }\end{array}$ \\
\hline & mc-Si & 30 & 1.9 & $\begin{array}{c}\eta_{1}=13.2 ; \eta_{2}=80 ; P R=24 \\
S R=1700-2280 ; T I=\text { roof-top; } \\
\text { GL = Europe, USA }\end{array}$ \\
\hline & CdTe & 19 & 0.7 & $\begin{array}{c}\eta_{1}=9 ; \eta_{2}=80 ; P R=24 ; \\
S R=1700-2280 ; T I=\text { roof-top; } \\
\text { GL = Europe, USA }\end{array}$ \\
\hline \multirow{3}{*}{ Held and Iig [32] } & CdTe & 29.5 & 1.1 & $\begin{array}{l}\eta_{1}=10.9 ; \eta_{2}=80 ; P R=200,000 ; \\
S R=1200 ; T I=\text { ground-mount; } \\
\text { GL = Central Europe (Germany) }\end{array}$ \\
\hline & CdTe & 20.9 & 0.76 & $\begin{array}{c}\eta_{1}=10.9 ; \eta_{2}=80 ; \mathrm{PR}=200,000 ; \\
\mathrm{SR}=1700 ; \mathrm{TI}=\text { ground-mount; } \\
\mathrm{GL}=\text { Mediterranean region, } \\
\text { Europe (Italy) }\end{array}$ \\
\hline & CdTe & 20.9 & 0.9 & $\begin{array}{c}\eta_{1}=10.9 ; \eta_{2}=80 ; P R=200,000 ; \\
S R=1700 ; T I=\text { ground-mount; } \\
\text { GL = Mediterranean region, } \\
\text { Europe }(E U-25)\end{array}$ \\
\hline
\end{tabular}


Table 1. Cont

\begin{tabular}{|c|c|c|c|c|}
\hline Source & $\begin{array}{c}\text { PV Category } \\
\text { (Supplementary Case } \\
\text { Description) }\end{array}$ & $\begin{array}{c}\text { GHG } \\
\text { Emissions } \\
\left(\mathrm{gCO}_{2} \mathrm{e} / \mathrm{kWh}\right)\end{array}$ & $\begin{array}{l}\text { EPBT } \\
\text { (Years) }\end{array}$ & $\begin{array}{c}\text { Additional Features } \\
\eta_{1}(\%), \eta_{2}(\%), \text { PR (kW), } \\
\text { SR }\left(\mathrm{kWh} / \mathrm{m}^{2} / \mathrm{yr}\right), \mathrm{TI}, \mathrm{GL}\end{array}$ \\
\hline \multirow{2}{*}{ Held and Iig [32] } & CdTe & 18.7 & 0.7 & $\begin{array}{c}\eta_{1}=10.9 ; \eta_{2}=80 ; P R=200,000 \\
S R=1900 ; T I=\text { ground-mount; } \\
\text { GL = Spain }\end{array}$ \\
\hline & CdTe & 18.7 & 0.9 & $\begin{array}{c}\eta_{1}=10.9 ; \eta_{2}=80 ; P R=200,000 \\
S R=1900 ; T I=\text { ground-mount; } \\
\text { GL = Portugal }\end{array}$ \\
\hline \multirow{3}{*}{$\begin{array}{l}\text { International } \\
\text { Energy Agency } \\
\text { Report [33] }\end{array}$} & sc-Si & 38 & 1.7 & $\begin{array}{c}\eta_{1}=14 ; \eta_{2}=75 ; \mathrm{SR}=1700 \\
\mathrm{TI}=\text { roof-top; } \\
\text { GL = Southern Europe }\end{array}$ \\
\hline & mc-Si & 34 & 1.7 & $\begin{array}{c}\eta_{1}=13.2 ; \eta_{2}=75 ; S R=1700 \\
\text { TI = roof-top; } \\
\text { GL = Southern Europe }\end{array}$ \\
\hline & CdTe & 18 & 0.8 & $\begin{array}{c}\eta_{1}=10.9 ; \eta_{2}=75 ; \mathrm{SR}=1700 \\
\mathrm{TI}=\text { roof-top; } \\
\mathrm{GL}=\text { Southern Europe }\end{array}$ \\
\hline Desideri et al. [34] & mc-Si & 88.74 & 4.17 & $\begin{array}{c}\eta_{1}=14.4 ; \eta_{2}=80 ; P R=1778 \\
T I=\text { ground-mount, } 25^{\circ} \text { tilt } \\
\text { GL }=\text { Perugia, Italy }\end{array}$ \\
\hline
\end{tabular}

Tilt of solar panels: due South unless specified. NA: not available. PR: power rating. SR: solar radiation. TI: type of installation. GL: geographical location.

\subsection{Review of CSP LCA Studies}

Many studies [35-46] evaluated the life cycle environmental impacts of using CSP electricity generation systems. The majority of the CSP studies [35,37,39-42,44-46] defined the LCA boundary conditions to include activities, such as manufacturing (extraction of raw materials, transportation to the manufacturing facility, component manufacturing processes, transportation of the final product to regional storage), construction (activities associated with site improvements, transporting components to the site, plant assembly), operation, and maintenance (manufacture of replacement components and their transportation to the site, water consumption in the power block and for mirror cleaning, fuel consumption in cleaning/maintenance vehicles, on-site natural gas combustion, electricity consumption from the regional power grid), dismantling (energy required to disassemble the major CSP plant systems), and disposal (energy required to transport demolition waste to the landfill, incinerator, recycling plant, or re-manufacturer and the energy required for final disposal). One study [38] limited the life cycle boundary conditions to include only material production.

Table 2 provides a summary of the CSP electricity generation systems (based on the type of working mechanism) along with the corresponding GHG emissions (in $\mathrm{gCO}_{2} \mathrm{e} / \mathrm{kWh}$ ) and EPBT periods (in years) for each of the reviewed CSP electricity generation LCA study. Table 2 also presents additional site-specific features that included power rating (PR, expressed in $\mathrm{kW}$ ), available solar radiation at the location (SR, expressed in $\mathrm{kWh} / \mathrm{m}^{2} / \mathrm{yr}$ ), and the geographical location (GL) for the installed CSP systems. Based on the review of 12 CSP electricity generation LCA studies (refer to Table 2), one may note that parabolic trough $(N=10)$ CSP electricity generation systems were greater in number compared to central receiver $(N=9)$, solar chimney $(N=3)$, paraboloidal dish $(N=2)$, and solar pond $(N=2)$ CSP electricity generation systems. 
Table 2. GHG emissions and EPBT periods for CSP electricity generation systems.

\begin{tabular}{|c|c|c|c|c|}
\hline Source & $\begin{array}{c}\text { CSP Category } \\
\text { (Supplementary Case } \\
\text { Description) }\end{array}$ & $\begin{array}{c}\text { GHG } \\
\text { Emissions } \\
\left(\mathrm{gCO}_{2} \mathrm{e} / \mathrm{kWh}\right)\end{array}$ & $\begin{array}{l}\text { EPBT } \\
\text { (Years) }\end{array}$ & $\begin{array}{c}\text { Additional Features } \\
\text { PR (kW), SR }\left(\mathrm{kWh} / \mathrm{m}^{2} / \mathrm{yr}\right), \\
\text { GL }\end{array}$ \\
\hline Kreith et al. [35] & Central receiver & 43 & NA & $\mathrm{PR}=100,000 ; \mathrm{GL}=\mathrm{USA}$ \\
\hline CRIEPI Report [36] & Central receiver & 213 & NA & $\mathrm{PR}=5000 ; \mathrm{GL}=$ Saijo, Japan \\
\hline Martin [37] & Parabolic trough & 166 & & $\mathrm{GL}=\mathrm{USA}$ \\
\hline \multirow{8}{*}{ Norton et al. [38] } & $\begin{array}{c}\text { Central receiver } \\
\text { (energy efficient materials) }\end{array}$ & 21 & NA & GL = Europe \\
\hline & $\begin{array}{c}\text { Central receiver } \\
\text { (conventional materials) }\end{array}$ & 48 & NA & GL = Europe \\
\hline & $\begin{array}{c}\text { Paraboloidal dish } \\
\text { (energy efficient materials) }\end{array}$ & 24 & NA & $\mathrm{GL}=$ Europe \\
\hline & $\begin{array}{c}\text { Paraboloidal dish } \\
\text { (conventional materials) }\end{array}$ & 58 & NA & $\mathrm{GL}=$ Europe \\
\hline & $\begin{array}{c}\text { Parabolic trough } \\
\text { (energy efficient materials) }\end{array}$ & 30 & NA & GL = Europe \\
\hline & $\begin{array}{c}\text { Parabolic trough } \\
\text { (conventional materials) }\end{array}$ & 80 & NA & GL = Europe \\
\hline & $\begin{array}{c}\text { Solar pond } \\
\text { (energy efficient materials) }\end{array}$ & 5 & NA & $\mathrm{GL}=$ Europe \\
\hline & $\begin{array}{c}\text { Solar pond } \\
\text { (conventional materials) }\end{array}$ & 6 & NA & $\mathrm{GL}=$ Europe \\
\hline \multirow{2}{*}{ Weinrebe et al. [39] } & Parabolic trough & 17 & NA & $\begin{array}{c}\mathrm{PR}=80,000 ; \mathrm{SR}=2300 \\
\mathrm{GL}=\text { California, } \mathrm{USA}\end{array}$ \\
\hline & Central receiver & 25 & NA & $\begin{array}{c}\mathrm{PR}=30,000 ; \mathrm{SR}=2300 \\
\mathrm{GL}=\text { California, USA }\end{array}$ \\
\hline \multirow{2}{*}{ Lenzen [40] } & Central receiver & 60 & NA & $\mathrm{SR}=2350 ; \mathrm{GL}=$ Australia \\
\hline & Parabolic trough & 90 & NA & $\mathrm{SR}=2350 ; \mathrm{GL}=$ Australia \\
\hline \multirow{2}{*}{ Lechon et al. [41] } & Parabolic trough & 185 & 1.04 & $\begin{array}{l}\mathrm{PR}=17,000 ; \mathrm{SR}=2016 ; \\
\mathrm{GL}=\text { Andalucía, Spain }\end{array}$ \\
\hline & Central receiver & 203 & 1.02 & $\begin{array}{l}\mathrm{PR}=50,000 ; \mathrm{SR}=1997 \\
\mathrm{GL}=\text { Andalucía, } \\
\text { Spain }\end{array}$ \\
\hline \multirow{2}{*}{ NEEDS Report [42] } & Parabolic trough & 161 & NA & SR = 2000; GL = Spain \\
\hline & Central receiver & 140 & NA & SR = 2000; GL = Spain \\
\hline Niemann et al. [43] & Solar chimney & 10 & NA & $\begin{array}{c}\mathrm{PR}=50 ; \mathrm{GL}=\text { Manzanares } \\
\text { Spain }\end{array}$ \\
\hline \multirow[b]{2}{*}{$\begin{array}{c}\text { Burkhardt et al. } \\
\text { [44] }\end{array}$} & $\begin{array}{l}\text { Parabolic trough (wet: use of } \\
\text { wet-cooling systems) }\end{array}$ & 26 & 1 & $\begin{array}{l}\mathrm{PR}=103,000 \mathrm{~kW} ; \mathrm{SR}=2700 \\
\quad \mathrm{GL}=\text { California, USA; }\end{array}$ \\
\hline & $\begin{array}{c}\text { Parabolic trough } \\
\text { (dry: elimination of } \\
\text { wet-cooling systems) }\end{array}$ & 28 & 1.08 & $\begin{array}{l}\mathrm{PR}=103,000 \mathrm{~kW} ; \mathrm{SR}=2700 \\
\quad \mathrm{GL}=\text { California, USA; }\end{array}$ \\
\hline \multirow{2}{*}{ Fabrizi [45] } & Parabolic trough & 15 & $<1$ & $\mathrm{GL}=\mathrm{USA}$ \\
\hline & Central receiver & 18 & $<1$ & $\mathrm{GL}=\mathrm{USA}$ \\
\hline \multirow{2}{*}{ Zongker [46] } & Solar chimney & 34.1 & NA & $\mathrm{GL}=\mathrm{USA}$ \\
\hline & Solar chimney & 62.9 & NA & $\mathrm{GL}=\mathrm{USA}$ \\
\hline
\end{tabular}

NA: not available.

\subsection{Statistical Evaluation of PV LCA Studies}

Figure 1 provides a graphical representation of the (a) error bars (mean $\pm 95 \%$ confidence interval (CI) statistics) and (b) box plots (quartiles + outlier statistics) for GHG emissions from the different PV electricity generation systems reviewed in this study. Table 3 provides a statistical summary of 
the life cycle GHG emissions with details on the sample size $(N)$, mean $(\mathrm{X}) \pm$ standard deviation (SD), minimum (Min.), maximum (Max.), standard error of the mean (SE), quartile 1 (Q1), quartile 2 or median (Q2), and quartile $3(\mathrm{Q} 3)$ for the different PV electricity generation systems reviewed in this study.

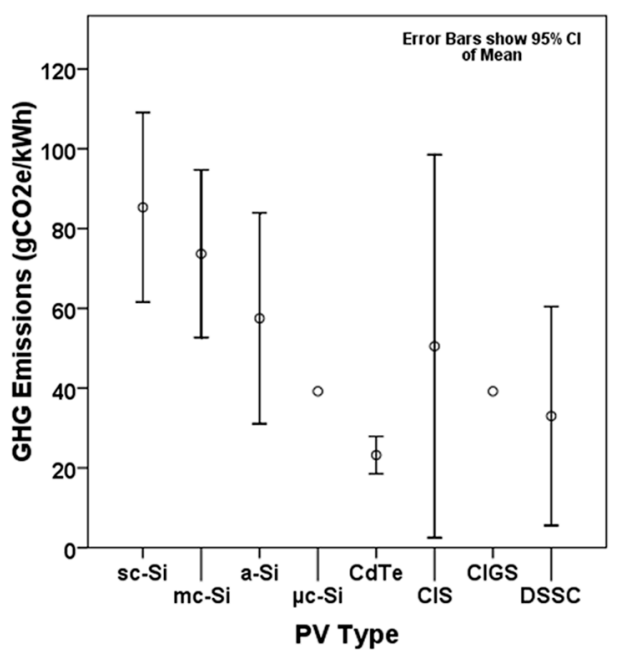

(a)

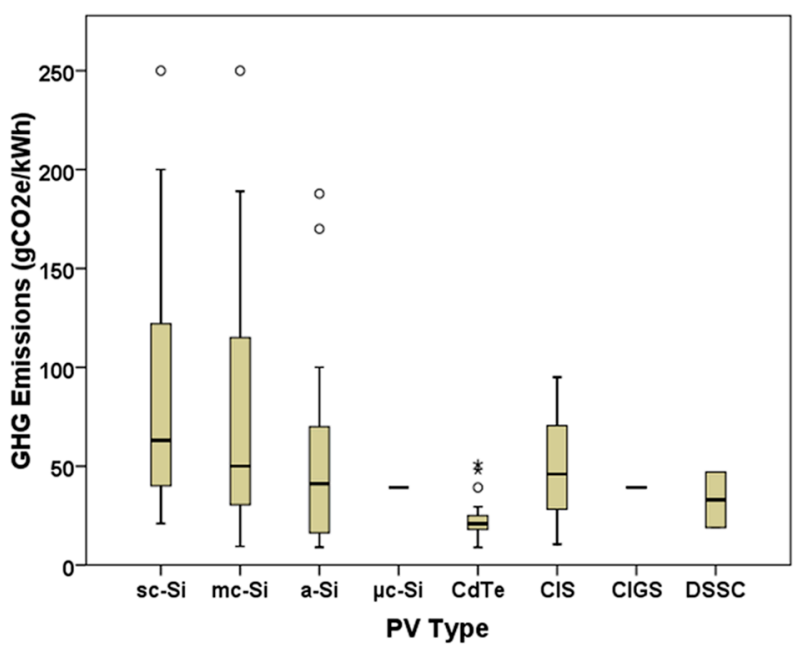

(b)

Figure 1. GHG emissions from PV electricity generation systems: (a) mean $\pm 95 \%$ CI error bars and (b) quartile box plots.

Table 3. GHG emission ( $\mathrm{gCO}_{2} \mathrm{e} / \mathrm{kWh}$ ) statistics from PV electricity generation systems.

\begin{tabular}{ccccccccc}
\hline PV Type & $\boldsymbol{N}$ & $\mathbf{X} \pm \mathbf{S D}$ & Min. & Max. & SE & Q1 & Q2 & Q3 \\
\hline sc-Si & 24 & $85.33 \pm 59.43$ & 21 & 250 & 12.13 & 38 & 63 & 114 \\
$\mathrm{mc}-\mathrm{Si}$ & 35 & $73.68 \pm 63.44$ & 9.4 & 250 & 10.72 & 30 & 50 & 120 \\
$\mathrm{a}-\mathrm{Si}$ & 16 & $57.49 \pm 53.96$ & 9 & 187.8 & 13.49 & 15 & 41.1 & 50 \\
$\mu \mathrm{c}-\mathrm{Si}$ & 1 & $39.2 \pm 0$ & 39.2 & 39.2 & 0 & 39.2 & 39.2 & 39.2 \\
$\mathrm{CdTe}$ & 21 & $23.22 \pm 10.94$ & 8.9 & 51 & 2.39 & 18 & 20.9 & 25 \\
$\mathrm{CIS}$ & 3 & $50.5 \pm 42.43$ & 10.5 & 95 & 24.5 & 10.5 & 46 & 95 \\
CIGS & 1 & $39.2 \pm 0$ & 39.2 & 39.2 & 0 & 39.2 & 39.2 & 39.2 \\
DSSC & 2 & $33 \pm 19.8$ & 19 & 47 & 14 & 19 & 33 & 47 \\
\hline
\end{tabular}

From Figure 1a and Table 3, one may note that the mean life cycle GHG emissions obtained from the use of sc-Si, mc-Si, a-Si, $\mu \mathrm{c}-\mathrm{Si}, \mathrm{CdTe}$, CIS, CIGS, and DSSC in PV electricity generation systems are $85.33 \mathrm{gCO}_{2} \mathrm{e} / \mathrm{kWh}, 73.68 \mathrm{gCO}_{2} \mathrm{e} / \mathrm{kWh}, 57.49 \mathrm{gCO}_{2} \mathrm{e} / \mathrm{kWh}, 39.2 \mathrm{gCO}_{2} \mathrm{e} / \mathrm{kWh}, 23.22 \mathrm{gCO}_{2} \mathrm{e} / \mathrm{kWh}$, $50.5 \mathrm{gCO}_{2} \mathrm{e} / \mathrm{kWh}, 39.2 \mathrm{gCO}_{2} \mathrm{e} / \mathrm{kWh}$, and $33 \mathrm{gCO}_{2} \mathrm{e} / \mathrm{kWh}$, respectively. These results indicate that lower GHGs are emitted from the organic and thin film non-organic material-based PV electricity generation systems in comparison to the widely adopted c-Si non-organic material-based PV electricity generation systems. The higher mean life cycle GHG emissions for sc-Si/mc-Si may be attributed to the fact that higher energy consumption (a fraction delivered by fossil fuels) is associated with the silicon purification and crystallization processes when manufacturing the sc-Si/mc-Si PV modules [47]. The mc-Si PV electricity generation systems produced less GHG emissions in comparison to sc-Si PV electricity generation systems. Similar observations were made by several other studies $[4,7,10,12,23,26,29,31,33,47]$. Among the thin film PV categories, a-Si PVs had the highest GHG emissions (attributed to silicon processing activities utilizing a fraction of fossil fuel energy). The CdTe material-based PV electricity generation systems produced the minimum GHG emissions among all the different material-based PV electricity generation system options considered in this study. Similar observations were made by a number of studies $[20,21,23,25,26,31,33]$. The lower GHG 
emissions from CdTe PV electricity generation systems in comparison to other PV electricity generation systems may be attributed to the recovery of CdTe from the waste modules [20]. The GHGs emitted by DSSC PV electricity generation systems were also found to be on the lower side. From Figure $1 \mathrm{~b}$, one may note the degree of variation in GHG emissions was less for thin film (a-Si, CdTe, CIS, CIGS) and organic (DSSC) PV electricity generation systems when compared to the c-Si (sc-Si, mc-Si) PV electricity generation systems. More LCA studies utilizing $\mu \mathrm{c}-\mathrm{Si}$ and CIGS PV electricity generation systems are to be considered before one generalizes the influence of $\mu \mathrm{c}-\mathrm{Si}$ and CIGS on the life cycle GHG emissions (note that the mean life cycle GHG emission statistics of $\mu \mathrm{c}-\mathrm{Si}$ and CIGS PVs in this study were based on a sample size equal to one).

Figure 2 provides a graphical representation of the (a) error bars (mean $\pm 95 \%$ confidence interval (CI) statistics) and (b) box plots (quartiles + outlier statistics) for EPBT periods from the different PV electricity generation systems reviewed in this study. Table 4 provides a statistical summary of the EPBT periods for the different PV electricity generation systems reviewed in this study. From Figure $2 a$ and Table 4, one may note that the mean EPBT period was found to be the highest for sc-Si PVs (6.07 years), followed by a-Si (3.18 years), mc-Si (3.15 years), $\mu \mathrm{c}-\mathrm{Si}$ /CIGS (2.5 years), CIS (2.07 years), and CdTe (1.25 years). These results indicate sc-Si PVs takes longer time periods for energy pay back in comparison to other PVs and the longer EPBT periods may be attributed to the additional energy inputs involving fossil fuel energy that are required for various silicon processing activities. Among the thin film PVs, a-Si PV had the highest EPBT period. This may be attributed as a consequence of the lower conversion efficiencies associated with it [47]. The lower EPBT periods for thin film PVs are associated with less raw materials and energy consumption than those required for c-Si PVs [47]. The median quartile statistic showed a consistent pattern with the mean EPBT periods from different PV electricity generation systems (refer to Figure $2 \mathrm{~b}$ and Table 4).

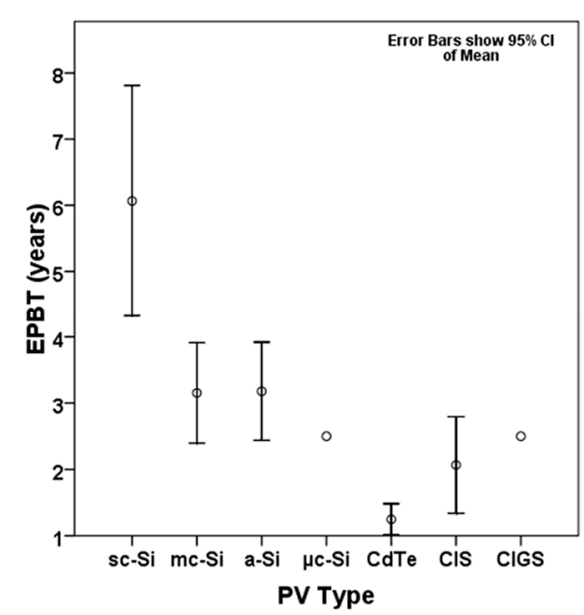

(a)

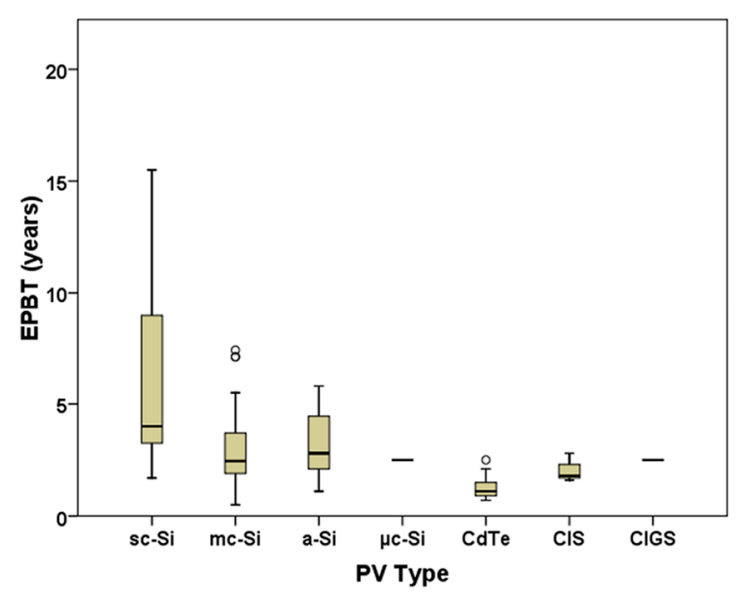

(b)

Figure 2. EPBT periods for PV electricity generation systems: (a) mean $\pm 95 \% \mathrm{CI}$ error bars and (b) quartile box plots.

$\eta_{2}$ represents the percentage energy exported to the grid after the deduction of energy losses and energy consumption for operation, thereby providing a qualitative measure to compare PV systems across different locations. Figure 3 provides a graphical representation of the (a) error bars (mean $\pm 95 \%$ confidence interval (CI) statistics) and (b) box plots (quartiles + outlier statistics) for $\eta_{2}$ of the different PV electricity generation systems reviewed in this study. Table 5 provides a statistical summary of $\eta_{2}$ for the different PV electricity generation systems reviewed in this study. From Figure 3a and Table 5, one may note the mean $\eta_{2}$ for sc-Si, mc-Si, a-Si, $\mu \mathrm{c}-\mathrm{Si}$, CdTe, CIS, CIGS, and DSSC to be $78.19 \%, 77.73 \%$, $79.16 \%, 75 \%, 78.49 \%, 76.87 \%, 75 \%$, and $53 \%$, respectively. These results indicate a relatively equivalent performance for all the PV electricity generation systems (75\%-78.3\%) with the exception of DSSC, 
which had a lower $\eta_{2}$ value. From Figure $3 b$, one may note the degree of variation in $\eta_{2}$ is less and approximately equivalent for all the material-based PV electricity generation systems reviewed in this study.

Table 4. EPBT period (years) statistics for PV electricity generation systems.

\begin{tabular}{ccccccccc}
\hline PV Type & $\boldsymbol{N}$ & $\mathbf{X} \pm \mathbf{S D}$ & Min. & Max. & SE & Q1 & Q2 & Q3 \\
\hline sc-Si & 19 & $6.07 \pm 3.88$ & 1.7 & 15.5 & 0.89 & 3.2 & 4 & 8.9 \\
mc-Si & 24 & $3.15 \pm 1.89$ & 0.5 & 7.4 & 0.39 & 1.9 & 2.4 & 3.6 \\
a-Si & 16 & $3.18 \pm 1.51$ & 1.1 & 5.8 & 0.38 & 2.1 & 2.8 & 4 \\
$\mu \mathrm{c}-S i$ & 1 & $2.5 \pm 0$ & 2.5 & 2.5 & 0 & 2.5 & 2.5 & 2.5 \\
CdTe & 18 & $1.25 \pm 0.51$ & 0.7 & 2.5 & 0.12 & 0.9 & 1.1 & 1.5 \\
CIS & 3 & $2.07 \pm 0.64$ & 1.6 & 2.8 & 0.37 & 1.6 & 1.8 & 2.8 \\
CIGS & 1 & $2.5 \pm 0$ & 2.5 & 2.5 & 0 & 2.5 & 2.5 & 2.5 \\
\hline
\end{tabular}

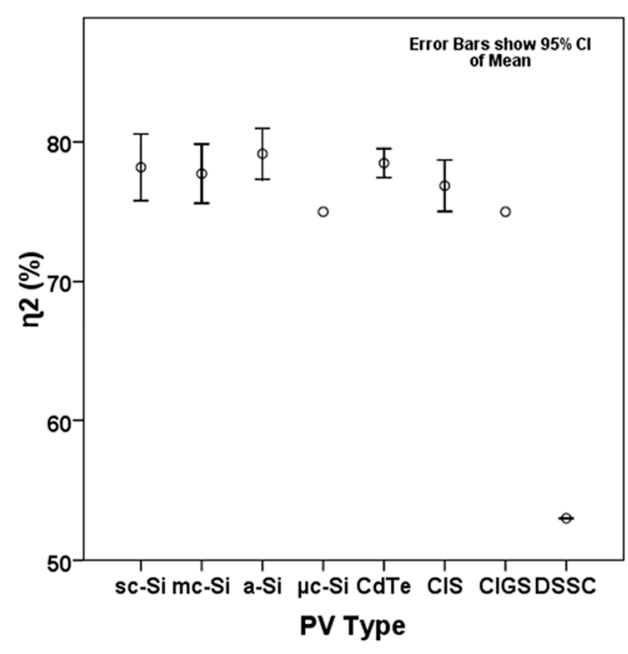

(a)

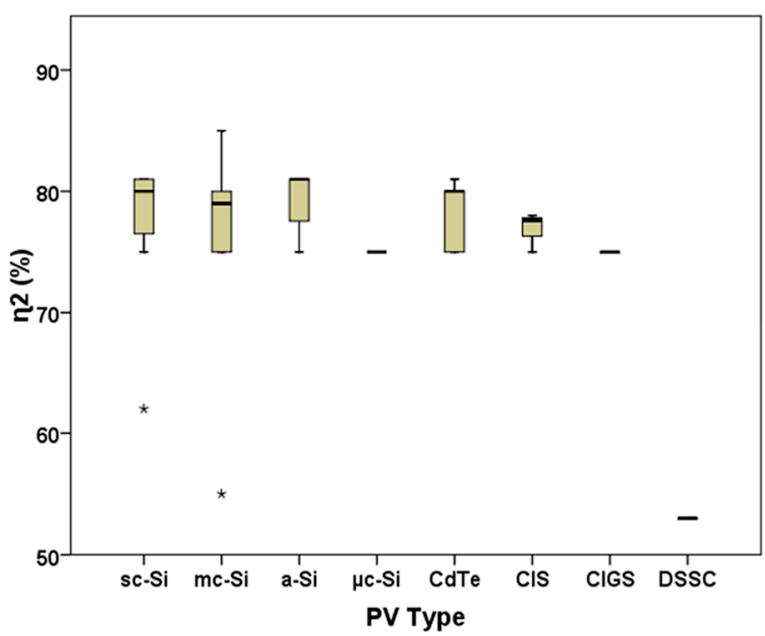

(b)

Figure 3. $\eta_{2}$ for different PV systems: (a) mean $\pm 95 \%$ CI error bars; (b) quartile box plots.

Table 5. $\eta_{2}(\%)$ statistics for PV electricity generation systems.

\begin{tabular}{ccccccccc}
\hline PV Type & $\boldsymbol{N}$ & $\mathbf{X} \pm \mathbf{S D}$ & $\mathbf{M i n}$. & Max. & SE & Q1 & Q2 & Q3 \\
\hline sc-Si & 16 & $78.19 \pm 4.88$ & 62 & 81 & 1.22 & 75 & 80 & 81 \\
mc-Si & 26 & $77.73 \pm 5.52$ & 55 & 85 & 1.08 & 75 & 79 & 80 \\
a-Si & 7 & $79.16 \pm 2.46$ & 75 & 81 & 0.93 & 77.1 & 81 & 81 \\
$\mu \mathrm{c}-S i$ & 1 & $75 \pm 0$ & 75 & 75 & 0 & 75 & 75 & 75 \\
CdTe & 21 & $78.49 \pm 2.42$ & 75 & 81 & 0.53 & 75 & 80 & 80 \\
CIS & 3 & $76.87 \pm 1.63$ & 75 & 78 & 0.94 & 75 & 77.6 & 78 \\
CIGS & 1 & $75 \pm 0$ & 75 & 75 & 0 & 75 & 75 & 75 \\
DSSC & 2 & $53 \pm 0$ & 53 & 53 & 0 & 53 & 53 & 53 \\
\hline
\end{tabular}

\subsection{Statistical Evaluation of CSP LCA Studies}

Figure 4 provides a graphical representation of the (a) error bars (mean $\pm 95 \%$ confidence interval (CI) statistics) and (b) box plots (quartiles + outlier statistics) for GHG emissions for the different CSP electricity generation systems reviewed in this study. Table 6 provides a statistical summary of the GHG emissions from different CSP electricity generation systems reviewed in this study. From Figure 4a and Table 6 one may note that the central receiver CSP electricity generation system had the highest mean GHG emissions $\left(85.67 \mathrm{gCO}_{2} \mathrm{e} / \mathrm{kWh}\right)$, followed by the parabolic trough 
(79.8 $\left.\mathrm{gCO}_{2} \mathrm{e} / \mathrm{kWh}\right)$, the paraboloidal dish $\left(41 \mathrm{gCO}_{2} \mathrm{e} / \mathrm{kWh}\right)$, the solar chimney $\left(35.67 \mathrm{gCO}_{2} \mathrm{e} / \mathrm{kWh}\right)$, and the solar pond $\left(5.5 \mathrm{gCO}_{2} \mathrm{e} / \mathrm{kWh}\right)$ electricity generation systems. These results indicate that the widely used central receiver and parabolic trough CSP electricity generation systems emitted more GHGs than the paraboloidal dish, the solar chimney, and the solar pond CSP electricity generation systems. The paraboloidal dish and the solar chimney CSP electricity generation systems produced approximately only $50 \%$ of the GHGs emitted by the paraboloidal dish and the central receiver CSP electricity generation systems. The solar pond CSP electricity generation system produced the least amount of GHG emissions. From Figure 4b, one may note the degree of variation in GHG emissions was less for the solar pond, the paraboloidal dish, and the solar chimney CSP electricity generation systems in comparison with the central receiver and the parabolic trough CSP electricity generation systems. The parabolic trough and the central receiver had approximately equivalent EPBT periods of one year (refer to Table 2). For the remaining three CSP electricity generation systems, there were no established data with regards to EPBT periods. While more LCA research is needed to analyze the performance of CSP electricity generation systems in general, priority needs to be given to analyzing the LCA of solar pond, paraboloidal dish, and solar chimney CSP electricity generation systems.

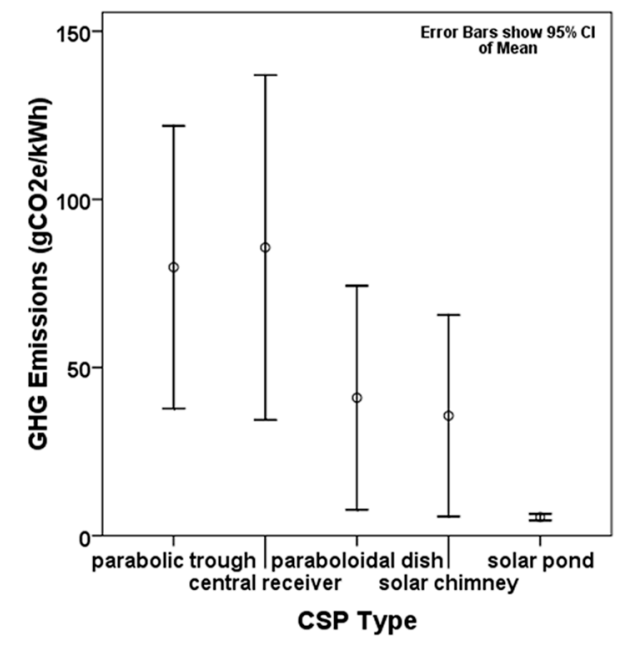

(a)

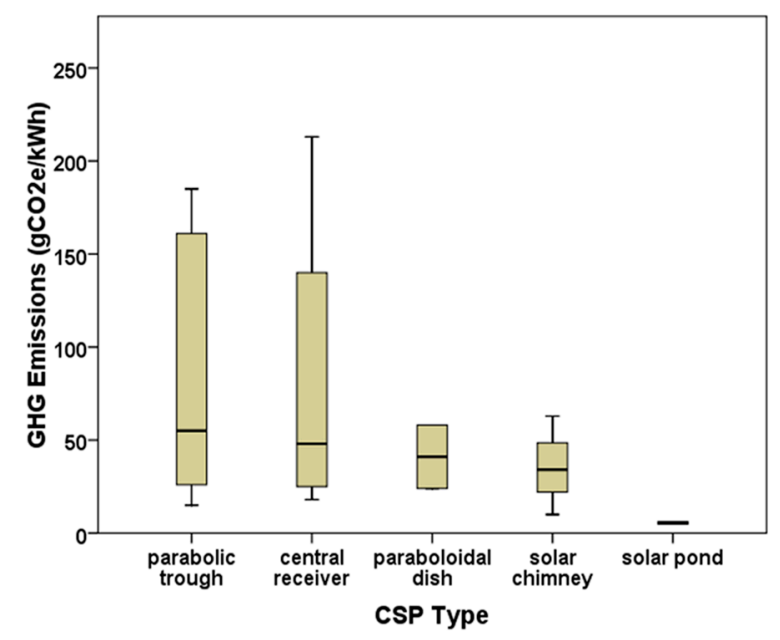

(b)

Figure 4. GHG emissions from CSP electricity generation systems: (a) mean $\pm 95 \%$ CI error bars and (b) quartile box plots.

Table 6. GHG emission $\left(\mathrm{gCO}_{2} \mathrm{e} / \mathrm{kWh}\right)$ statistics from CSP electricity generation systems.

\begin{tabular}{ccccccccc}
\hline CSP Type & $\boldsymbol{N}$ & $\mathbf{X} \pm \mathbf{S D}$ & Min. & Max. & SE & Q1 & Q2 & Q3 \\
\hline parabolic trough & 10 & $79.8 \pm 67.82$ & 15 & 185 & 21.45 & 26 & 55 & 161 \\
central receiver & 9 & $85.67 \pm 78.48$ & 18 & 213 & 26.16 & 25 & 48 & 140 \\
paraboloidal dish & 2 & $41 \pm 24.04$ & 24 & 58 & 17 & 24 & 41 & 58 \\
solar chimney & 3 & $35.67 \pm 26.49$ & 10 & 62.9 & 15.29 & 10 & 34.1 & 62.9 \\
solar pond & 2 & $5.5 \pm 0.71$ & 5 & 6 & 0.5 & 5 & 5.5 & 5 \\
\hline
\end{tabular}

\section{Conclusions}

This paper examined the life cycle GHG emissions from all of the currently available material-based PV (sc-Si, mc-Si, a-Si, $\mu \mathrm{c}-\mathrm{Si}$, CdTe, CIS, CIGS, DSSC) and the working mechanism-based CSP (parabolic trough, central receiver, paraboloidal dish, solar chimney, solar pond) electricity generation systems. The life cycle GHG emissions from PV and CSP electricity generation systems were examined in this study by performing a review of the PV and CSP LCA studies (with categorization of all the identified PV and CSP LCA studies) and the subsequent computation of statistical parameters. 
A review of the literature on solar energy related LCA studies showed that there are numerous studies that evaluated the life cycle GHG emissions of PVs and there are only a limited number of studies that analyzed the life cycle GHG emissions of CSPs. A total of 31 PV LCA case studies and 12 CSP LCA case studies were reviewed in this study.

The mean life cycle GHG emissions from sc-Si, mc-Si, a-Si, $\mu \mathrm{c}-\mathrm{Si}$, CdTe, CIS, CIGS, and DSSC PV electricity generation systems were computed to be $85.33 \mathrm{gCO}_{2} \mathrm{e} / \mathrm{kWh}, 73.68 \mathrm{gCO}_{2} \mathrm{e} / \mathrm{kWh}$, $57.49 \mathrm{gCO}_{2} \mathrm{e} / \mathrm{kWh}, 39.2 \mathrm{gCO}_{2} \mathrm{e} / \mathrm{kWh}, 23.22 \mathrm{gCO}_{2} \mathrm{e} / \mathrm{kWh}, 50.5 \mathrm{gCO}_{2} \mathrm{e} / \mathrm{kWh}, 39.2 \mathrm{gCO}_{2} \mathrm{e} / \mathrm{kWh}$, and $33 \mathrm{gCO}_{2} \mathrm{e} / \mathrm{kWh}$, respectively. Prior PV LCA studies identified the level of solar radiation, position of modules, modules manufacturing energy intensity, and corresponding fuel mix, and solar radiation conversion efficiency to be the major factors influencing the life cycle performance of PVs. The mc-Si $\mathrm{PVs}$ proved to be a better cost-effective and environmental-friendly option than sc-Si PVs (considering the relatively lower GHG emissions and lesser EPBT period for an approximately equivalent $\eta_{2}$ value of $78 \%$ ). Thin-film PVs may be recommended for a wider implementation due to their positive attributes of emitting less GHGs and lower EPBT periods in comparison with c-Si PVs. The lower GHG emissions and EPBT periods for thin-films are associated with less raw materials and energy consumption. Amongst all of the different categories of PVs considered in this study, the most environmentally friendly option is the CdTe PV system (lowest GHG emission and EPBT periods for $\eta_{2}$ value of $78.49 \%$ ). While no representative LCA studies were noted in the literature for the OPV electricity generation systems, only a single LCA case study to represent $\mu \mathrm{c}-\mathrm{Si}$, DSSC PV electricity generation systems. More research efforts are needed to study the LCA of organic material based PV electricity generation systems.

The mean life cycle GHG emissions for the parabolic trough, central receiver, paraboloidal dish, solar chimney, and solar pond CSP electricity generation systems were computed to be $79.8 \mathrm{gCO}_{2} \mathrm{e} / \mathrm{kWh}, 85.67 \mathrm{gCO}_{2} \mathrm{e} / \mathrm{kWh}, 41 \mathrm{gCO}_{2} \mathrm{e} / \mathrm{kWh}, 35.67 \mathrm{gCO}_{2} \mathrm{e} / \mathrm{kWh}$, and $5.5 \mathrm{gCO}_{2} \mathrm{e} / \mathrm{kWh}$, respectively. The widely-used parabolic trough and central receiver CSP electricity generation systems emitted approximately 50\% more GHGs than the paraboloidal dish, solar chimney, and solar pond CSP electricity generation systems. Future CSP LCA studies need to focus more on analyzing the environmental contributions from the paraboloidal dish, solar chimney, and solar pond electricity generation systems, for which there were limited references.

Acknowledgments: This work was supported by the US National Science Foundation through the CREST Center for Energy and Environmental Sustainability (CEES) at Prairie View A\&M University, award number 1036593. The authors would like to thank Dr. Venkata Botlaguduru for his assistance with the revisions of the manuscript.

Author Contributions: Raghava Kommalapati conceived the idea and initiated the LCA work on different energy technologies. Akhil Kadiyala scrutinized the solar PV and CSP electricity generation system life cycle GHG emissions in-detail, with assistance from Md. Tarkik Shahriar. Professor Huque participated in many discussions.

Conflicts of Interest: The authors declare no conflict of interest.

\section{References}

1. International Energy Statistics. Available online: http://www.eia.gov/cfapps/ipdbproject/iedindex3.cfm? tid=44\&pid=44\&aid=2\&cid=ww,r1,\&syid=2008\&eyid=2012\&unit=QBTU (accessed on 9 November 2015).

2. International Energy Outlook 2013. Available online: http://www.eia.gov/forecasts/ieo/pdf/0484(2013).pdf (accessed on 9 November 2015).

3. Annual Energy Outlook 2015 with Projections to 2040. Available online: http:/ / www.eia.gov / forecasts / aeo/pdf/0383\%282015\%29.pdf (accessed on 9 November 2015).

4. Schaefer, H.; Hagedorn, G. Hidden energy and correlated environmental characteristics of PV power generation. Renew. Energy 1992, 2, 159-166. [CrossRef]

5. Environmental Life-Cycle Assessment of Multicrystalline Silicon Solar Cell Modules. Available online: https:/ / stuff.mit.edu/afs/athena/dept/cron/project/urban-sustainability/Old\%20files\%20from\% 20summer\%202009/Bjorn/solar/LCA\%20silicon\%20solar\%20cell\%20modules\%201995.pdf (accessed on 21 December 2014). 
6. Nieuwlaar, E.; Alsema, E.; Van Engelenburg, B. Using life-cycle assessments for the environmental evaluation of greenhouse gas mitigation options. Energy Convers. Manag. 1996, 37, 831-836. [CrossRef]

7. Kato, K.; Murata, A.; Sakuta, K. An evaluation on the life cycle of photovoltaic energy system considering production energy of off-grade silicon. Sol. Energy Mat. Sol. Cells 1997, 47, 95-100. [CrossRef]

8. Dones, R.; Frischknecht, R. Life cycle assessment of photovoltaic systems: Results of Swiss studies on energy chains. Prog. Photovolt. Res. Appl. 1998, 6, 117-125. [CrossRef]

9. Frankl, P.; Masini, A.; Gamberale, M.; Toccaceli, D. Simplified life-cycle analysis of PV systems in buildings: Present situation and future trends. Prog. Photovolt. Res. Appl. 1998, 6, 137-146. [CrossRef]

10. Kato, K.; Murata, A.; Sakuta, K. Energy pay-back time and lifecycle $\mathrm{CO}_{2}$ emission of residential PV power system with silicon PV module. Prog. Photovolt. Res. Appl. 1998, 6, 105-115. [CrossRef]

11. PV-BILD: A Life Cycle Environmental and Economic Assessment Tool for Building-Integrated Photovoltaic Installations. Available online: http://css.snre.umich.edu/publication/pv-bild-life-cycle-environmentaland-economic-assessment-tool-building-integrated (accessed on 21 December 2014).

12. Alsema, E.A. Energy pay-back time and $\mathrm{CO}_{2}$ emissions of PV systems. Prog. Photovolt. Res. Appl. 2000, 8, 17-25. [CrossRef]

13. Oliver, M.; Jackson, T. The evolution of economic and environmental cost for crystalline silicon photovoltaics. Energy Policy 2000, 28, 1011-1021. [CrossRef]

14. Greijer, H.; Karlson, L.; Lindquist, S.E.; Hagfeldt, A. Environmental aspects of electricity generation from a nanocrystalline dye sensitized solar cell system. Renew. Energy 2001, 23, 27-39. [CrossRef]

15. Kato, K.; Hibino, T.; Komoto, K.; Ihara, S.; Yamamoto, S.; Fujihara, H. A life-cycle analysis on thin-film CdS/CdTe PV modules. Sol. Energy Mat. Sol. Cells 2001, 67, 279-287. [CrossRef]

16. Nomura, N.; Inaba, A.; Tonooka, Y.; Akai, M. Life-cycle emission of oxidic gases from power-generation systems. Appl. Energy 2001, 68, 215-227. [CrossRef]

17. Life-Cycle Assessment of Electricity Generation Systems and Applications for Climate Change Policy Analysis. Available online: http:/ fti.neep.wisc.edu/pdf/fdm1181.pdf (accessed on 21 December 2014).

18. Ito, M.; Kato, K.; Sugihara, H.; Kichimi, T.; Song, J.; Kurokawa, K. A preliminary study on potential for very large scale photovoltaic power generation (VLS-PV) system in the Gobi desert from economic and environmental viewpoints. Sol. Energy Mat. Sol. Cells 2003, 75, 507-517. [CrossRef]

19. Fthenakis, V.M.; Kim, H.C. Energy use and greenhouse gas emissions in the life cycle of thin film CdTe photovoltaics. In Proceedings of the Symposium G-Life Cycle Analysis, MRS Fall Meeting, Boston, MS, USA, 28-30 November 2005.

20. Alsema, E.A.; de Wild-Scholten, M.J.; Fthenakis, V.M. Environmental impacts of PV electricity generation-A critical comparison of energy supply options. In Proceedings of the 21st European Photovoltaic Solar Energy Conference, Dresden, Germany, 4-8 September 2006.

21. Fthenakis, V.M.; Alsema, E. Photovoltaics energy payback times, greenhouse gas emissions and external costs: 2004-early 2005 status. Prog. Photovolt. Res. Appl. 2006, 14, 275-280. [CrossRef]

22. Kannan, R.; Leong, K.C.; Osman, R.; Ho, H.K.; Tso, C.P. Life cycle assessment study of solar PV systems: An example of a $2.7 \mathrm{kWp}$ distributed solar PV system in Singapore. Sol. Energy 2006, 80, 555-563. [CrossRef]

23. Fthenakis, V.M.; Kim, H.C. Greenhouse-gas emissions from solar electric- and nuclear power: A life-cycle study. Energy Policy 2007, 35, 2549-2557. [CrossRef]

24. Pacca, S.; Sivaraman, D.; Keoleian, G.A. Parameters affecting the life cycle performance of PV technologies and systems. Energy Policy 2007, 35, 3316-3326. [CrossRef]

25. Raugei, M.; Bargigli, S.; Ulgiati, S. Life cycle assessment and energy pay-back time of advanced photovoltaic modules: CdTe and CIS compared to poly-Si. Energy 2007, 32, 1310-1318. [CrossRef]

26. Fthenakis, V.M.; Kim, H.C.; Alsema, E. Emissions from photovoltaic life cycles. Environ. Sci. Technol. 2008, 42, 2168-2174. [CrossRef]

27. Ito, M.; Kato, K.; Komoto, K.; Kichimi, T.; Kurokawa, K. A comparative study on cost and life-cycle analysis for $100 \mathrm{MW}$ very large-scale PV (VLS-PV) systems in deserts using m-Si, a-Si, CdTe, and CIS modules. Prog. Photovolt. Res. Appl. 2008, 16, 17-30. [CrossRef]

28. García-Valverde, R.; Miguel, C.; Martínez-Béjar, R.; Urbina, A. Life cycle assessment study of a $4.2 \mathrm{kWp}$ stand-alone photovoltaic system. Sol. Energy 2009, 83, 1434-1445. [CrossRef]

29. Ito, M.; Komoto, K.; Kurokawa, K. Life-cycle analyses of very-large scale PV systems using six types of PV modules. Curr. Appl. Phys. 2010, 10, S271-S273. [CrossRef] 
30. Bravi, M.; Parisi, M.L.; Tiezzi, E.; Basosi, R. Life cycle assessment of a micromorph photovoltaic system. Energy 2011, 36, 4297-4306. [CrossRef]

31. Fthenakis, V.M.; Kim, H.C. Photovoltaics: Life-cycle analyses. Sol. Energy 2011, 85, 1609-1628. [CrossRef]

32. Held, M.; Iig, R. Update of environmental indicators and energy payback time of CdTe PV systems in Europe. Prog. Photovolt. Res. Appl. 2011, 19, 614-626. [CrossRef]

33. Life Cycle Inventories and Life Cycle Assessments of Photovoltaic Systems. Available online: https://www. bnl.gov/pv/files/pdf/226_Task12_LifeCycle_Inventories.pdf (accessed on 21 December 2014).

34. Desideri, U.; Proietti, S.; Zepparelli, F.; Sdringola, P.; Bini, S. Life cycle assessment of a ground-mounted 1778 kWp photovoltaic plant and comparison with traditional energy production systems. Appl. Energy 2012, 97, 930-943. [CrossRef]

35. Kreith, F.; Norton, P.; Brown, D. A comparison of $\mathrm{CO}_{2}$ emissions from fossil and solar power plants in the United States. Energy 1990, 15, 1181-1198. [CrossRef]

36. Central Research Institute of Electric Power Industry (CRIEPI). 1995 Annual Research Report. Energy Technology Life Cycle Analysis that Takes $\mathrm{CO}_{2}$ Emission Reduction into Consideration; CRIEPI: Tokyo, Japan, 1995.

37. Martin, J.A. A total fuel cycle approach to reducing greenhouse gas emissions: Solar generation technologies as greenhouse gas offsets in U.S. utility systems. Sol. Energy 1997, 59, 195-203. [CrossRef]

38. Norton, B.; Eames, P.C.; Lo, S.N.G. Full-energy-chain analysis of greenhouse gas emissions for solar thermal electric power generation systems. Renew. Energy 1998, 15, 131-136. [CrossRef]

39. Weinrebe, G.; Bohnke, M.; Trieb, F. Life cycle assessment of an 80MWSEGS plant and a 30MWPHOEBUS power tower. In Proceedings of the 1998 ASME International Solar Energy Conference 'Solar Engineering', Albuquerque, NM, USA, 14-17 June 1998; ASME: New York, NY, USA, 1998; pp. 417-424.

40. Lenzen, M. Greenhouse gas analysis of solar-thermal electricity generation. Sol. Energy 1999, 65, 353-368. [CrossRef]

41. Lechon, Y.; de la Rúa, C.; Sáez, R. Life cycle environmental impacts of electricity production by solar thermal power plants in Spain. J. Sol. Energy Eng. 2008, 130, 021012. [CrossRef]

42. Final Report on Technical Data, Costs, and Life Cycle Inventories of Solar Thermal Power Plants. Available online: http://www.needs-project.org/RS1a/RS1a\%20D12.2\%20Final\%20report\% 20concentrating\%20solar\%20thermal\%20power\%20plants.pdf (accessed on 3 June 2015).

43. Niemann, H.J.; Lupi, F.; Hoeffer, R.; Hubert, W.; Borri, C. The solar updraft power plant: Design and optimization of the tower for wind effects. In Proceedings of the 5th European and African Conference on Wind Engineering EACWE5, Florence, Italy, 19-23 July 2009.

44. Burkhardt, J.J.; Heath, G.A.; Turchi, C.S. Life cycle assessment of a parabolic trough concentrating solar power plant and the impacts of key design alternatives. Environ. Sci. Technol. 2011, 45, 2457-2464. [CrossRef] [PubMed]

45. CSP Technologies, Environmental Impact 2012. Available online: https://www.esmap.org/sites/esmap. org/files/ESMAP_IFC_RE_CSP_Training_World\%20Bank\%20Fabrizi.pdf (accessed on 3 June 2015).

46. Zongker, J.D. Life Cycle Assessment of Solar Updraft Tower Power Plant: EROEI and GWP as a Design Tool. Master's Thesis, Wichita State University, Wichita, KS, USA, 2013.

47. Peng, J.; Lu, L.; Yang, H. Review on life cycle assessment of energy payback and greenhouse gas emission of solar photovoltaic systems. Renew. Sustain. Energy Rev. 2013, 19, 255-274. [CrossRef]

48. Amponsah, N.W.; Troldborg, M.; Kington, B.; Aalders, I.; Hough, R.L. Greenhouse gas emissions from renewable energy sources: A review of lifecycle considerations. Renew. Sustain. Energy Rev. 2014, 39, 461-475. [CrossRef]

49. IPCC Special Report on Renewable Energy Sources and Climate Change Mitigation. Available online: http:/ / srren.ipcc-wg3.de/report (accessed on 21 December 2014).

(C) 2017 by the authors. Licensee MDPI, Basel, Switzerland. This article is an open access article distributed under the terms and conditions of the Creative Commons Attribution (CC BY) license (http:/ / creativecommons.org/licenses/by/4.0/). 